\title{
Linx
}

Revue des linguistes de l'université Paris X Nanterre

$54 \mid 2006$

La cause : approche pluridisciplinaire

\section{La causalité d'après la psychologie sociale cognitive : les explications naïves du comportement}

\section{Ahogni N'Gbala}

\section{(2) OpenEdition}

1 Journals

Édition électronique

URL : http://journals.openedition.org/linx/509

DOI : $10.4000 /$ linx.509

ISSN : 2118-9692

Éditeur

Presses universitaires de Paris Nanterre

\section{Édition imprimée}

Date de publication : 1 juin 2006

Pagination : 113-124

ISSN : 0246-8743

\section{Référence électronique}

Ahogni N'Gbala, «La causalité d'après la psychologie sociale cognitive : les explications naïves du comportement », Linx [En ligne], 54 | 2006, mis en ligne le 01 août 2007, consulté le 10 décembre 2020. URL : http://journals.openedition.org/linx/509 ; DOI : https://doi.org/10.4000/linx.509 


\title{
La causalité d'après la psychologie sociale cognitive : les explications naives du comportement ${ }^{1}$
}

\author{
Abogni N'Gbala, Université de Paris $X$ - Nanterre \\ Laboratoire de Psychologie Sociale (EA 1588)
}

\section{Introduction}

Pour commencer, considérons le comportement de ces deux adolescents :

- «Paul, 13 ans, retrouve dans les vestiaires du collège un portefeuille égaré contenant 100 euros, les documents d'identité et carte bleue du propriétaire; il empoche l'argent et laisse le portefeuille où il l'a trouvé ».

- «Georges, 13 ans, retrouve dans les vestiaires du collège un portefeuille égaré contenant 100 euros, les documents d'identité et carte bleue du propriétaire. Il dépose le portefeuille au secrétariat pour restitution au propriétaire ».

Pourquoi Paul et Georges ont-ils agi ainsi ?

La psychologie naïve ou psychologie du sens commun permet de répondre à cette question. En effet, je voudrais parler non pas de l'explication du comportement par le psychologue, mais plutôt de l'explication naïve du comportement telle qu'étudiée

\footnotetext{
${ }^{1}$ Je voudrais remercier ici le professeur Thierry Meyer du Laboratoire de Psychologie Sociale (EA 1588) qui a bien voulu lire et commenter la première version de cet exposé, Julien Chappé et Alice Follenfant pour leur aide à la préparation formelle de ce travail, et bien d'autres personnes qui à des degrés divers y ont contribué. Je remercie également les organisateurs de cette journée pour l'opportunité qu'ils m'ont donnée de communiquer à un public pluridisciplinaire sur l'approche de la psychologie sociale cognitive concernant la causalité naïve.
} 
Ahogni N'gbala

par la psychologie scientifique, notamment, la psychologie sociale cognitive et expérimentale. En d'autres termes, je voudrais donner l'éclairage de la psychologie scientifique sur la psychologie du sens commun ou psychologie naïve.

La psychologie naïve ou psychologie du sens commun est l'explication quotidienne du comportement. Cette psychologie est constituée des connaissances "empiriques", de savoirs culturels sur le pourquoi et les conséquences du comportement. Elle préexiste à la psychologie scientifique constituée des connaissances obtenues par le biais de méthodes rigoureuses telles que l'observation systématique et l'expérimentation. Opposables à plusieurs égards, ces deux types de psychologies cohabitent pacifiquement et s'interpénètrent (Kelley, 1992 ; Moscovici, 1976).

Mon propos s'articulera autour des points suivants : (1) «L'étude de la psychologie du sens commun » qui servira à présenter brièvement les motivations des psychologues sociaux pour l'étude de la psychologie du sens commun; 2) «Les causes dans l'explication naïve» qui décrira les différentes caractéristiques de la cause dans l'explication naïve; 3) «Les conséquences des attributions causales » permettra de montrer comment l'attribution causale influence en retour les réactions émotionnelles et cognitives (prédictions) du percevant face à l'événement observé ; 4) «Comment fait-on des attributions causales? » où seront brièvement présentés quelques uns des modèles théoriques qui décrivent les processus cognitifs sous-tendant l'inférence causale ; 5) «Applications des théories de l'attribution causale » pour montrer qu'il est possible de partir des causes que le sujet place à l'origine des événements qu'il vit pour l'aider à atteindre un mieux être.

Mais avant de poursuivre, je voudrais donner quelques définitions. L'expression attribution causale sera utilisée pour désigner l'explication quotidienne parce que faire une attribution, c'est inférer une cause pour expliquer un événement. Le terme événement désignera le comportement, l'action observée ou effectuée (aller au cinéma; danser), ou le résultat de cette action (réussir à un examen). Les termes acteur et sujet percevant (ou observateur) désigneront, respectivement, la personne dont on essaie d'expliquer le comportement et la personne qui donne les explications.

\section{L'étude de la psychologie du sens commun}

L'étude de la psychologie du sens commun commence avec Fritz Heider (1958), un psychologue social. Dans son ouvrage, "The psychology of Interpersonal Relations », Heider livre ses «insight» sur les analogies entre la fonction et le mode de construction des explications quotidiennes et la démarche scientifique d'appréhension des événements. Cette vision peut se résumer dans les deux citations suivantes :

Il est un principe pour la psychologie du sens commun, comme de la théorie scientifique en général, que l'homme parvienne à une maitrise de la réalité, qu'il puisse la prédire et la contrôler, en reliant les comportements et événements 
variables qu'il observe quotidiennement à des conditions relativement inchangeables sous-jacentes, à savoir, les soi- disant propriétés dispositionnelles de l'environnement. (Heider, 1958, p. 79)

Pour la psychologie du sens commun (comme pour la psychologie scientifique) le résultat de l'action serait dû au jeu de deux groupes de conditions, notamment, les facteurs liés à la personne et les facteurs liés à l'environnement (Heider, 1958, p. 82).

Ainsi, l'homme de la rue et l'homme de science seraient non seulement motivés par le même besoin de comprendre et de prédire les événements, mais ils procèderaient de la même façon pour atteindre cet objectif. En outre, ils auraient les mêmes conceptions sur l'origine du comportement. Ces similitudes font de l'homme de la rue, un « scientifique intuitif » ou naif.

En quoi la démarche que suit l'homme de la rue pour arriver à expliquer les événements se rapproche-t-elle de celle du scientifique? Pour les psychologues sociaux, un début de réponse se trouve dans les propositions faites par les «théories de l'attribution causale ${ }^{2} »$. On appelle théories de l'attribution causale l'ensemble des modèles théoriques suscités par les hypothèses de Heider (1958) et dont l'objectif est de décrire les processus par lesquels le sujet naif construit ses explications, comment ses explications influencent en retour ses réactions émotionnelles ou cognitives vis-àvis de l'événement ou des personnes qui en sont à l'origine, mais aussi quelles difficultés il rencontre face à la complexité même du phénomène d'explication.

\section{Les causes dans l'explication naïve}

L'explication commence par l'identification de la cause de l'événement. Ainsi, vous aurez expliqué le comportement de Paul décrit plus haut si vous dites qu'il a agi comme il l'a fait parce qu'il est «malhonnête » et celui de Georges, en disant qu'il est «honnête». Sachez cependant que pour les théories de l'attribution, ces deux explications contradictoires sur le plan de la moralité supposée des deux garçons sont équivalentes sur un certain nombre de points. En effet, les théoriciens de l'attribution causale ont une façon bien à eux d'approcher les causes évoquées dans une explication. Celles-ci sont catégorisées selon des caractéristiques appelées dimensions.

D’après Weiner et collaborateurs, les auteurs du «modèle attributionnel de la motivation à l'accomplissement et de l'émotion » dont on parlera davantage ci-dessous, les causes varient sur trois dimensions (Weiner, 1979 ; 1985). La première est le lieu de causalité. En référence à l'idée de Heider (1958) selon laquelle les causes du comportement seraient liées à des caractéristiques de la personne ou de l'environnement, le lieu de causalité renvoie à la localisation de la cause. Ainsi, l'attribution causale est dite interne

\footnotetext{
2 Il s'agira d'une présentation brève des théories de l'attribution. Le lecteur désireux d'en savoir plus sur ces théories consultera avantageusement les revues de questions en anglais et en français qui y ont été consacrées.
} 
Abogni N'gbala

lorsqu'elle porte sur quelque chose de la personne (autrui ou soi-même) - l'acteur dont on essaie de comprendre le comportement; cela peut porter sur des dispositions, aptitudes, ou traits de personnalité de l'acteur, de son état de santé, de fatigue, ou d'humeur. Au contraire, l'attribution est dite externe lorsqu'elle renvoie à un aspect de la situation, l'environnement physique ou social; il peut s'agir par exemple de la difficulté/facilité d'une tâche, du bon/mauvais temps, des normes sociales, des dispositions, l'état de santé et l'humeur des autres, etc. La deuxième dimension causale est la contrôlabilité. Cela renvoie au degré d'influence qu'on attribue à l'individu, ou que celui-ci croit pouvoir exercer sur les causes à l'origine de son comportement. La cause est dite «contrôlable» quand l'acteur se sent capable de la modifier (niveau d'effort, motivation) ou est perçu comme tel ; elle sera dite «incontrôlable » si elle est jugée audessus des forces de l'acteur, ou indépendante de sa volonté (normes sociales, niveau d'intelligence). La troisième dimension est la stabilité, c'est-à-dire, le degré de permanence de la cause ; la cause est dite «stable » lorsqu'elle est jugée permanente et susceptible de se maintenir dans le temps (trait de personnalité, niveau d'intelligence) ou « instable » quand elle est de nature passagère ou temporaire (effort, (mal)chance). En résumé, on peut expliquer la réussite de quelqu'un à un examen en disant que c'est parce qu'il est «doué», parce qu'il a «fait des efforts», parce que "l'examen était facile » ou parce qu'il « s'est fait aider par un ami ». «Doué » et « fait des efforts » sont deux causes internes, mais la première est stable et incontrôlable par l'acteur, tandis que la seconde est instable et contrôlable ; la «facilité » de l'examen et « se faire aider » sont des causes externes dont la première est stable et incontrôlable par l'acteur, tandis que la seconde est instable et contrôlable.

A ces trois premières dimensions s'ajoute une quatrième, la globalité, qui renvoie au degré de généralisation de la cause (Seligman et collaborateurs, 1979). La cause est dite " globale » lorsqu'on s'attend à ce qu'elle occasionne les événements dans diverses situations, ou «spécifique », lorsque que son impact est limité à un événement précis. Evoquer l'intelligence (ou la stupidité) pour expliquer la bonne (mauvaise) performance d'un élève à un examen de math serait une cause globale dans la mesure où cela laisse supposer que cet élève est susceptible de réussir (d'échouer) non seulement aux examens en math, mais dans d'autres matières également (physiques, SVT, etc.); en revanche, attribuer ces mêmes performances en maths au fait que cet élève est « doué/peu doué pour les maths » serait faire une attribution spécifique dans la mesure où cela limite les effets de cette cause au seul domaine des maths.

\section{Conséquences des attributions causales}

Les attributions causales ont des conséquences sur la personne qui les fait, mais aussi sur les personnes et événements à propos desquels elles sont faites; il s'agit des réponses ou réactions émotionnelles (colère, joie, etc.), cognitives (perception sociale, prédiction, etc.) ou comportementales (aide/refus d'aide à autrui) du sujet percevant. Le modèle attributionnel de la motivation à l'accomplissement et de l'émotion de Weiner et collaborateurs a permis de systématiser l'étude des conséquences des attributions causales. 
Considérons ces deux cas : deux élèves ont eu $5 / 20$ et échoué à un examen de math. L'un ressent beaucoup de tristesse et ne s'attend pas à réussir à un examen similaire dans le futur ; au contraire, l'autre ressent peu de tristesse et s'attend à réussir à un examen de même type. Qu'est-ce qui détermine ces réactions apparemment paradoxales de ces élèves au même résultat ? Il est clair que la valence des résultats explique dans une certaine mesure les réactions émotionnelles de nos deux élèves, mais pas du tout leurs attentes. C'est du reste la position de Weiner et collaborateurs qui pensent que les dimensions des attributions faites par les élèves pour expliquer leurs résultats rendraient suffisamment compte de la spécificité de leurs réactions.

Le lieu de causalité déterminerait les réactions émotionnelles liées à l'estime de soi (sentiment de compétence, fierté, honte): un échec fera moins honte à l'acteur s'il l'attribue à une cause externe (la malchance, la difficulté de l'examen) qu'à une cause interne (n'avoir pas fait d'effort, manque d'habileté); la stabilité, ainsi que la contrôlabilité, déterminerait les attentes du sujet: l'acteur s'attendrait à échouer la prochaine fois s'il attribue son échec présent à une cause stable (manque d'habileté, difficulté de l'examen) que s'il l'attribue à une cause instable (la malchance, n'avoir pas fait d'effort); l'habileté, la difficulté de l'examen et la malchance étant incontrôlables par l'acteur, celui-ci s'attendra à réussir s'il attribue son échec à une cause contrôlable (manque d'effort). En somme, de nos deux élèves, on peut penser que le premier a fait une attribution interne (i.e., manque d'habileté) ou externe (i.e., difficulté de l'examen) stable et incontrôlable pour son échec, tandis que l'autre a fait une attribution interne, instable et contrôlable (manque d'effort) ou externe, instable, incontrôlable (la malchance).

\section{Comment fait-on les attributions causales?}

Les théories de l'attribution offrent plusieurs réponses à cette question (Hewstone, 1989). Je me contenterai de présenter ici les deux qui représentent des positions contradictoires en apparence, mais complémentaires dans le fond. La première, c'est que l'homme de la rue formerait ses attributions causales « en traitant des informations ». L'idée que le traitement d'informations serait le passage obligé pour faire une attribution causale découle en fait de l'hypothèse selon laquelle le sujet naïf est un scientifique intuitif. Les modèles qui privilégient cette hypothèse mettent l'accent sur les informations en question, leur mode de traitement et les explications auxquelles cela permet d'aboutir. Deux de ces modèles sont présentés ci-dessous.

La seconde réponse, c'est que l'homme de la rue formerait ses attributions causales «en se basant sur des heuristiques ». Cette hypothèse repose sur le constat d'échec de la première. En effet, on se rendra compte à la pratique que le sujet naïf a tendance à s'écarter des modèles "normatifs » d'attribution causale basés sur le traitement d'informations. Cela va amener à réviser à la baisse l'importance initialement accordée au traitement des informations dans le processus d'explication naïve, mais aussi la perception initiale du sujet naï. Ainsi, il a été conclu que celui-ci 
Abogni N'gbala

est en fait un «avare cognitif» (cognitive miser, Fiske \& Taylor, 1991) qui préfère les raccourcis ou heuristiques au traitement coûteux d'informations. Je commenterai brièvement quelques unes de ces approches privilégiant l'économie cognitive dans l'attribution causale.

\subsection{Première réponse : «en traitant des informations "}

4.1.1. Le modèle de la covariation (Kelley, 1967)

Le plus populaire de ces modèles est celui de la covariation de Kelley (1967). Covariation signifie « apparition simultanée de deux faits ou phénomènes » : par exemple, tabagisme et cancer du poumon; effort au travail et réussite. C'est au philosophe J. S. Mill que Kelley emprunte l'idée que la covariation est une méthode qui permet de connaittre la cause des événements. En effet, d'après les observations du philosophe, «l'effet (l'événement observé) est attribué à la cause qui est présente quand l'effet est présent, et absente quand l'effet est absent».

Un exemple permettra d'illustrer comment Kelley conçoit la mise en œuvre du principe de la covariation pour faire une attribution causale. Imaginons que nous voulons expliquer le comportement de Jean qui a bien aimé le dernier film de Steven Spielberg. En terme d'attribution causale, il s'agit de savoir si la réaction de Jean au film est due à une cause interne (quelque chose qui serait lié à Jean) ou à une cause externe (quelque chose qui serait lié au film).

Selon Kelley, pour appliquer le principe de la covariation, le sujet naiff se servira de trois types particuliers d'informations. Il s'agit en premier lieu des informations sur le consensus. Celles-ci renseignent sur le comportement des autres gens (i.e., les autres spectateurs) qui ont été exposés au même stimulus (i.e., le film) que l'acteur. Le deuxième type d'informations concernées ici portent sur la différenciation (ou distinctivité) ; elles renseignent sur la réaction de l'acteur face à des stimulus de même ordre (i.e., les autres films). Enfin, seront prises en compte les informations sur la constance (ou consistance); elles renseignent sur la réaction de l'acteur face au même stimulus en diverses autres occasions et circonstances (i.e., sa réaction quand il a vu le film seul ou en présence d'amis, au cinéma ou à la télévision). Ces trois types d'informations-clés peuvent prendre soit un niveau élevé ou fort, soit un niveau bas ou faible. Ainsi, selon que la plupart des spectateurs ou peu d'entre eux, dans notre exemple, ont aimé le film, le consensus sera dit « fort » ou «faible ». La différenciation sera forte si Jean n'aime que ce film particulier du réalisateur, ou faible si Jean aime la plupart de ses films. La constance sera également forte si Jean aime le film quelles que soient les circonstances, ou faible quand il a aimé de l'avoir vu au cinéma mais pas à la télé, en présence d'amis mais pas lorsqu'il était seul.

D’après Kelley (1967), l'attribution dépend en général de la combinaison de ces informations selon leur niveau fort ou faible. Cette combinaison se fait intuitivement mais en suivant le plan rigoureux de l'analyse de la variance (Anova). Ainsi : 
- la combinaison Haut Consensus (la plupart des spectateurs aiment le film), Haute Différenciation (Jean n'aime que ce seul film du réalisateur) et Haute Constance (Jean aime le film quelles que soient les circonstances) pousse vers une attribution à l'entité, c'est-à-dire une attribution externe : c'est un bon film.

- la combinaison Bas Consensus (peu de spectateurs aiment le film), Haute Constance (Jean aime le film quelles que soient les circonstances), et Basse Différenciation (Jean aime tous les films du réalisateur) pousse vers une attribution à la personne, c'est-à-dire, une attribution interne : une caractéristique propre à Jean fait qu'il aime ce film.

\subsubsection{La théorie des inférences correspondantes (Jones \& Davis, 1965)}

La théorie des inférences correspondantes de Jones et Davis (1965) est chronologiquement le premier modèle théorique proposé pour tester empiriquement les hypothèses de Heider (1958) concernant l'homme de la rue comme un scientifique intuitif. La position seconde attribuée à ce modèle dans cet exposé tient du fait d'une part qu'il est relativement moins populaire que celui de la covariation de Kelley (1967), et d'autre part, du fait qu'il ne porte pas de façon directe sur les inférences causales (Hewstone, 1989). J'en parle ici pour illustrer davantage l'idée de traitement d'informations.

La théorie des inférences correspondantes rend compte de la manière par laquelle le sujet naïf arrive à inférer des dispositions stables à l'origine du comportement qu'il observe. L'inférence de disposition suit deux étapes clés :

- Étape 1 : le sujet naïf doit établir au préalable que l'acteur avait l'intention de se comporter tel qu'il a fait en répondant aux questions suivantes : l'individu était-il capable d'avoir le comportement en question et avait-il une claire conscience de ses conséquences?

Partons de l'événement suivant: un étudiant en Ile de France désireux de faire carrière en psychologie s’inscrit à Paris V plutôt qu'à Paris X. A partir du moment où on sait que cet étudiant remplit bien les conditions pour être accepté à Paris V, et qu'il connaît les exigences de travail de l'établissement, on dira qu'il avait bien l'intention de s'y inscrire.

- Etape 2 : Pour inférer la disposition (l'étudiant aime bien la psychologie et veut en faire sa carrière) qui sous-tend l'intention, le sujet percevant devra répondre à trois autres questions sur:

- la spécificité des effets liés à l'événement (les universités Paris X, Paris XIII pouvaient-elles permettre à l'étudiant d'atteindre ses objectifs de carrière ou seule Paris $\mathrm{V}$ le pouvait ?) ;

- la présence ou non de désirabilité sociale (l'étudiant de Paris V est-il socialement plus valorisé, plus respecté que celui des autres universités ?) ;

- la liberté de choix (l'étudiant pouvait-il librement s'inscrire dans l'une des autres universités ou était-il contraint de ne s'inscrire qu'à Paris V ?).

S'il apparait que seule Paris V peut garantir à l'étudiant la formation qu'il désire, qu’il n'y a aucun prestige à s'inscrire à Paris $\mathrm{V}$, et que l'étudiant était libre de ses choix, alors on peut lui attribuer sans risque de se tromper la disposition correspondante à son comportement. 
Abogni N'gbala

\subsubsection{Limites de la première réponse}

La vision qui fait du sujet naïf un scientifique intuitif ne sera que partiellement confirmée par une longue et abondante recherche sur les deux modèles théoriques décrits ci-dessus (voir Fiske \& Taylor, 1991 ; Hewstone, 1989, pour revues). En effet, contrairement aux prédictions du modèle de la covariation (Kelley, 1967), il apparaitra que les informations sur le consensus sont peu utilisées par le sujet naiff et que celui-ci s'intéresse à des informations ne figurant pas dans le modèle (Hewstone, 1989).

Les études portant sur la théorie des inférences correspondantes remettent également en question bien de ses présupposés. Je ne présenterai brièvement ici que les résultats des travaux ayant manipulé la liberté de choix. Dans ces études, l'acteur (un compère de l'expérimentateur) est amené à prendre devant un public composé des participants à l'expérience, une position sur un sujet polémique, par exemple, la peine de mort. Dans la condition liberté de choix, l'acteur se présente seul devant le public et expose librement les arguments en faveur de sa position (disons, favorable à la peine de mort); dans la condition contrainte, l'expérimentateur demande à l'acteur devant le public de prendre une position favorable à la peine de mort et lui fournit les arguments écrits pour cela. Ce sont les mêmes arguments que pour l'acteur prétendument libre. Les résultats montrent de façon systématique que les participants attribuent à l'acteur libre comme à l'acteur sous contrainte des dispositions justifiant la position qu'ils ont prises (voir Gilbert et Malone, 1995; Gilbert, 1998; Jones, 1990 pour revues). En clair, contrairement aux hypothèses de Jones et Davis (1965) la liberté de choix (présence/absence de contrainte) ne semble pas être une information prise en compte par le sujet percevant.

En somme, il est ressorti de l'ensemble des études que le sujet percevant non seulement ne prend pas en compte les informations, mais il ne les traite pas comme préconisé par les modèles théoriques; ceci le conduit à faire des attributions causales qui s'écartent des prédictions des modèles. Ces attributions non conformes aux modèles sont dites « erronées » ou « biaisées ». Par exemple, on a observé chez le sujet percevant une tendance systématique à faire des attributions internes ou dispositionnelles, minimisant ainsi la part de la situation ou de l'environnement dans l'apparition de l'événement. Ce type de réponse a reçu le nom «d'erreur fondamentale d'attribution» (Ross, 1977). On a également relevé chez le sujet une tendance à faire des attributions internes pour ses comportements positifs, mais des attributions externes pour ses comportements négatifs. Par exemple, un élève obtient 18 sur 20 en math et l'attribue à ses aptitudes dans la matière; il obtient $05 / 20$ à un examen analogue et l'attribue à la difficulté de l'épreuve, ou à l'incompétence de l'enseignant qui explique mal les leçons. Ces attributions asymétriques sont appelées «biais d'autocomplaisance » (Miller \& Ross, 1975 ; Zuckerman, 1979).

\subsection{Deuxième réponse : "en appliquant des heuristiques"}

Cette autre approche des processus d'attribution causale a été matérialisée par le développement de modèles d'usage plus économique pour le sujet. C’est le cas du 
« schéma de causalité » proposé par Kelley (1972) comme alternative au modèle de la covariation qui est une procédure «idéale » ne pouvant être appliquée que si l'on est assez motivé à investir du temps et de l'énergie pour obtenir les informations requises. Or, tel n'est pas toujours le cas. Aussi Kelley (1972) décrit-il une procédure plus économique, à savoir, les « schémas de causalité » auxquels l'on se référerait pour faire des attributions à propos des événements de la vie courante. Un «schéma de causalité est une connaissance générale que possède la personne à propos de la façon dont certaines causes interagissent pour produire certains types d'effets» (Kelley, 1972, p. 15). En d'autres termes, le schéma causal est la connaissance des relations entre causes et effets que nous tirons de notre expérience vécue et de nos apprentissages sociaux. On sait, par exemple, que « conduire en état d'ivresse cause des accidents ».

Une variante du modèle des «schémas de causalité » est le modèle de la «structure de connaissance » qui renvoie également aux connaissances empiriques que possède l'individu sur son environnement et les relations entre les différents éléments qui le composent (Lalljee \& Abelson, 1983). Selon les auteurs de cet autre modèle, les actions ne sont pas perçues de façon isolée par le sujet mais plutôt comme actions reliées les unes aux autres en une sorte d'unité (Read, 1987). Par exemple, le sujet percevant essaiera d'expliquer et de comprendre l'événement « Richard demande à son oncle de lui prêter de l'argent», en inférant que celui-ci veut réaliser un projet pour lequel il n'a pas suffisamment d'argent, que l'oncle a beaucoup d'argent et qu'il a l'habitude de prêter à Richard. Ces inférences sont non seulement spontanées, mais elles se basent sur des schémas d'interprétation des événements pour lesquels les informations relatives au modèle de la covariation ne sont ni disponibles, ni nécessaires. En outre, l'application des structures de connaissance permet à l'individu d'arriver à une explication de l'événement en termes concrets.

\section{Applications des théories de l'attribution}

Les attributions causales jouent un rôle dans la façon dont l'individu s'adapte à sa maladie physique (paralysie) et fait face au stress (Roesch \& Weiner, 2001). Elles donnent lieu à des applications pratiques en termes d'aide thérapeutique apportée au sujet (Dweck, 1975). La formation à la réattribution, initiée par Försterling (1985), est un mode d'intervention thérapeutique inspiré du modèle attributionnel de Weiner (1985) et d'autres qui n'ont pas été présentés dans l'exposé sur les théories (voir Vallerand, 1994). Ce mode d'intervention s'adresse aux personnes vivant un échec scolaire ou relationnel qu'elles se sentent incapables d'y remédier seules. Son principe de base repose sur l'idée que le comportement autant que les affects et croyances relatives à une situation sont des conséquences des attributions. En clair, si un individu confronté à un échec donné s'en trouve très affecté, démotivé et pessimiste quant aux chances de mieux faire dans une situation analogue dans le futur, c'est à cause des «mauvaises » attributions qu'il fait. Comme on l'a vu plus haut, l'attribution d'un échec à une cause interne, stable et incontrôlable (manque d'habileté) est capable de provoquer ces états chez l'individu. Il va donc s'agir d'amener l'individu vivant un échec chronique à faire des attributions plus « favorables » pour lui-même. 
Ahogni N'gbala

D'autres applications se font dans le domaine des thérapies cognitives. Par exemple, à des joueurs invétérés de loterie qui souhaitent limiter leur propension à jouer, le psychologue propose de faire des attributions externes (c'est le hasard) plutôt qu'internes (je suis mauvais aujourd'hui) chaque fois que l'envie de jouer les prend. Mû par le besoin de contrôler ses comportements, le joueur sera plus enclin à inhiber son envie de jouer attribuée à un facteur externe qui le dominerait.

\section{Conclusion}

La question que je posais pour commencer mon exposé était «En quoi la démarche que suit l'homme de la rue pour arriver à expliquer les événements se rapproche-t-elle de celle du scientifique? En d'autres termes, peut-on établir des analogies véritables entre les attributions causales (l'explication naïve) et l'explication scientifique »? La réponse qui puisse être faite au terme de cet exposé, c'est qu'il n'y a pas d'analogie entre le sujet naif et le scientifique. Pour preuve, les causes qu'évoque le sujet naïf pour expliquer les événements du quotidien ne résultent pas nécessairement d'une démarche scientifique. En effet, celui-ci manifeste une certaine paresse mentale qui l'amène à préférer les heuristiques; de fait, il ne traite pas les informations disponibles comme le prédisent les scientifiques, et commet des « erreurs » ou biaise ses explications.

Mais, cette réponse pourrait être amendée à la lumière des deux observations suivantes :

- les causes évoquées par le sujet naïf pour expliquer le comportement ont des dimensions particulières qui affectent, en retour, la façon dont il se perçoit ou perçoit les autres, ses anticipations et émotions ;

- les attributions causales jouent un rôle fondamental dans la vie de la personne au point qu'elles peuvent servir de base pour des thérapies.

La question alternative qui pourrait se poser serait de savoir: le fait que le psychologue scientifique puisse partir des attributions causales pour concevoir des approches thérapeutiques ne confère-t-il pas aux attributions causales et, partant, à la démarche qui les sous-tend, une certaine scientificité ? 


\section{RÉFÉRENCES BIBLIOGRAPHIQUES}

DWECK, C., 1975, «The role of expectations and attributions in the alleviation of learned helplessness», Journal of Personality and Social Psychology, 36: 951-962.

FiSKE, S.T., TAYLOR, S.E., 1991, Social cognition (2nd ed.), New York, McGraw-Hill.

FÖRSTERLING, F., «Attributional retraining: A review», Psychological Bulletin, 98: 495-512.

GilberT, D. T., 1998, «Ordinary personology», in D. T. Gilbert, S. T. Fiske, \& G. Lindzey (Eds.), The handbook of social psychology, 4th ed., Vol. 2, New York, McGraw-Hill: 89-150.

HEIDER, F., 1958, The psychology of interpersonal relations. New York, Wiley.

Hewstone, M., 1990, Causal attribution: From cognitive processes to collective beliefs. Oxford, UK: Blackwell.

JONES, E. E., 1990, Interpersonal perception. New York: Freeman.

JonES, E. E., DAvIS, K. E., 1965, «From acts to dispositions: The attribution process in person perception», in L. Berkowitz (Ed.), Advances in Experimental Social Psychology, Vol. 2, New York, Academic Press: 219-266.

KASSIN, S. M., PRYOR, J. B., 1985, «The development of attribution processes», in J. B. Pryor \& J. Day (Eds.), The development of social cognition, New York, Springer-Verlag: 3-34.

Kelley, H.H.,1967, «Attribution in social psychology», in D. Levine (Ed.), Nebraska Symposium on Motivation, Vol. 15, Lincoln: University of Nebraska Press, pp. 192-2380.

KELLEY, H.H., 1972, «Causal schemata and the attribution process», in E.E. Jones, D.E. \& Kelley, H. H., 1992, Common sense psychology and scientific psychology. Annual Review of Psychology, 43: 1-23.

MCARTHuR, L. A., 1972, «The how and what of why: Some determinants and consequences of causal attribution», Journal of Personality and Social Psychology, 22: 171-193.

Miller, D. T., \& Ross, M., 1975, «Self-serving biases in the attribution of causality: Fact or fiction?», Psychological Bulletin, 82: 213-225.

Moscovici, S., 1976, La psychanalyse, son image et son public. Paris, Presses Universitaires de France. 2ème Edition.

Roesch, S. C., \& Weiner, B., 2001, A meta-analytic review of coping with illness : Do causal attributions matter? Journal of Psychosomatic Research, 50: 205-219.

Seligman, M. E. P., Abramson, L. Y., Semmel, A., \& Von Baeyer, C., 1979, «Depressive attributional style», Journal of Abnormal Psychology, 88: 242-247.

VALLERAND, R. J., 1994, « Les attributions en psychologie sociale », in R. J. Vallerand (Ed.), Les fondements de la psychologie sociale. Québec, Gaëtan Morin: 259-326.

WEINER, B., 1979, «A theory of motivation for some classroom experiences», Journal of Educational Psychology, 71: 3-25. 
Abogni N'gbala

WEINER, B., 1985, «An attribution theory of achievement motivation and emotion», Psychological Review, 92: 548-573.

WeINER, B., 1995, Judgments of responsibility: A foundation for a theory of social conduct. New York, Guilford Press. 